\title{
Rubber compounds made of reactivated EPDM for fiber-reinforced elastomeric isolators: an experimental study
}

\author{
Ahmad Basshofi Habieb ${ }^{1} \cdot$ Federico Milani $^{2} \cdot$ Gabriele Milani $^{3}\left[\right.$ - Renato Cerchiaro ${ }^{4}$
}

Received: 26 April 2020 / Accepted: 15 August 2020 / Published online: 2 September 2020

(C) The Author(s) 2020

\begin{abstract}
Rubber recycling technology is a popular issue in many research fields, considering the huge amount of rubber waste in the environment. This paper discusses an application of regenerated ethylene propylene diene monomer (EPDM) to produce vulcanized items such as fiber-reinforced elastomeric isolators (FREIs), which are nowadays considered efficient low-cost seismic protection devices for low rise buildings (e.g., made by masonry) in developing countries. Two types of regenerated EPDM are studies and blended with two different virgin rubbers, Vistalon 3666 and Dutral 4038. The first virgin rubber is used to produce a compound with a hardness of around 30 Shore A, while the latter exhibits 60 Shore A. The present study, which is part of a wider research project aimed at the production of low cost un-bonded seismic isolation devices, focuses exclusively on the determination of both crosslinking degree through rheometer tests and elasticity/mechanical properties of the rubber pads, before and after ageing (hardness, tensile strength, elongation-at-break, stretch-stress behavior before and after ageing). The results show that the compounds with the second reactivated EPDM (type B) exhibit the most satisfactory performance, before and after ageing. This paper discusses also the method of fabrication of FREIs, obtained by the interposition of pads made by the selected recycled rubber and dry glass fiber-reinforced polymer (GFRP) textiles. The hardness tests performed on the sliced FREI specimen indicate that the vulcanization temperature used in the production is roughly suitable to obtain the expected rubber properties.
\end{abstract}

Keywords Rubber recycling $\cdot$ Reactivated EPDM $\cdot$ Fiber-reinforced elastomeric isolators (FREIs) $\cdot$ GFRP $\cdot$ Experimental characterization $\cdot$ Rheometer curves

\section{Introduction}

Rubber material is widely used either for household or industrial needs. Since the prehistoric era, rubber has been involved in human life by exploiting the latex from particular trees. In the industrial sector, natural rubber was first used at the beginning of the 18th century [1]. Nowadays,

Gabriele Milani

gabriele.milani@polimi.it

1 Department of Civil Engineering, Institut Teknologi Sepuluh Nopember, Jalan Teknik Kimia, 60111 Surabaya, Indonesia

2 Chem. Co Consultant, Via J.F. Kennedy 2, 45030 Occhiobello, Rovigo, Italy

3 Department of Architecture, Built Environment and Construction Engineering, Politecnico di Milano, Piazza Leonardo, 20133 Milano, Italy

4 Dergom S.R.L, Via dei Castagni, 3/5, 23846 Garbagnate Monastero, Lecco, Italy rubber is commonly used for tires, marine fenders, vibration or seismic isolation, impermeable layers, and also for sports equipment. In general, rubber can be in the form of natural and synthetic materials. A natural rubber can be traditionally harvested in the form of latex from the rubber tree. The latex is then refined into a rubber sheet ready for commercial processing. Thailand and Indonesia so far are the leading natural rubber producing countries in the world. Later on, synthetic rubber was introduced to overcome a high demand for rubber materials starting in the 1900 s due to a massive production of vehicles; [2]. Some of the popular synthetic rubber types include hypalon (CSM), ethylene propylene diene monomer (EPDM), viton (FPM), neoprene (CR), silicone rubber (MVQ), and styrene butadiene rubber (SBR).

To be ready for application, rubber should be processed through several stages; mixing or milling, and vulcanization [3]. In the mixing process, some additives are added to obtain the expected behavior of the rubber compound. In the vulcanization process, the rubber is heated with sulfur, 
accelerators and activators at around $140-160{ }^{\circ} \mathrm{C}$. The process triggers the formation of cross-links between long rubber molecules, thus improving tensile strength, hardness, and weather resistance.

Rubber waste recycling is nowadays one of the most important emergencies in terms of sustainability, considering that many industries download a huge amount of rubber waste in the environment. When dealing with the problem of rubber recycling, waste rubber can be reduced to powder and melt in blends with thermoplastic resins to produce thermoplastic elastomeric (TPE) compounds. As a matter of fact, the utilization of waste EPDM, for instance, appears very interesting, because EPDM backbone remains essentially the same as the starting materials. Numerous studies have been conducted to evaluate the application of recycled rubbers in various methods. Regenerated rubber seems to exhibit indeed a surprisingly good capacity of crosslinking; this is certainly a property known by the tire industry, which is taking its advantage and trying to recycle part of the waste in different contexts. To corroborate such conclusion, the filler amount (i.e., carbon black concentration) remains essentially unchanged.

Gregori et al. [4] investigated the effects of partial replacement of natural concrete aggregates with waste tire rubber on the concrete compressive strength. The proposed material, so called as rubberized concrete has mechanical and rheological properties suitable for civil engineering applications and leads to an effective solution of recycling discarded tires. However, rubberized concrete remains a suitable material mostly for nonstructural component such as lightweight wall panels, insulating screeds, and filling materials due to the high uncertainty of the predicted strength reduction factor.

An investigation was conducted in [5] on the flexural behavior of reinforced beams made from mixes of crumbed rubber concrete (CRC) where the beam specimens were tested up to failure. The results in this study show that the flexural behavior of reinforced CRC beam is very similar to the normal concrete beam of the same strength. It is also found in [6] that the ultimate shear capacities of CRC beams are $0-15 \%$ lower than that of an idealized beam of conventional concrete.

An innovative seismic isolation method was proposed by means of recycled scrap tire rubber-soil mixtures [7]. It is found that the proposed method can reduce the shaking level of ground motion not only in horizontal direction but also in vertical direction. Such a method can be considered as a lowcost solution for seismic protection of buildings, particularly in developing countries.

In the production of rubber base isolation systems, the use of recycled rubber has attracted high interest from researchers. The recycled rubber derived from used tires and industrial waste was used to produce rubber pads for fiber-reinforced elastomeric isolators (FREIs) [8]. Unlike the commercial base isolators, the proposed FREIs can be applied in un-bonded condition $[9,10]$, in which the upper and bottom surface of the isolator are not bonded to the superstructure and foundation. Therefore, the presence of steel end-plate is not required, reducing the construction cost of the isolation system. In addition, the un-bonded application may increase the deformability capacity and damping ratio of the isolator [11]. A test on shaking table [12,13] showed that the FREI system can effectively reduce the top displacement and the acceleration response of an isolated structure, competing with the available commercial devices. A successive research [14], conducted by combining the recycled rubber isolation system with additional magnetorial damper, showed how the seismic performance of the isolated structure again severe earthquakes is improved significantly. The cost of the recycled rubber isolators using fiber reinforcement is estimated about one-tenth of those of commercial isolators.

Scrap tire rubber pads (STRP) are used as alternative seismic base isolation systems [15, 16]. Additional steel shims or fiber laminas are not required because the reinforcing cords provided in tire manufacturing can be considered as the vertical reinforcement. The STRP layers are just stacked one on top of another without applying the adhesive. These proposed STRP isolators have several advantages compared to conventional elastomeric isolators including superior damping properties, lower cost, and easily available material.

A simple seismic isolation system has been proposed in [17] by simply using some half pieces of recycled tire applied in a particular configuration. Such a simple isolation system is designed to isolate critical rooms in health care facilities such as emergency rooms and essential care units. The experimental test shows that the proposed system demonstrates a feasible solution for improving the seismic response of non-structural components in critical rooms of hospital facilities.

The above research findings reveal the remarkable benefits of utilizing rubber waste from vehicle tires or industries both to drop down the cost of isolation system and to save the environment. Such a consideration is particularly essential when dealing with seismic protection in developing countries where the cost of the commercial isolation system is considered too expensive for massive application.

The present study investigates the use of recycled rubber in the form of reactivated ethylene propylene diene monomer (EPDM) from industrial waste to fabricate rubber pads for low-cost seismic isolation systems. The approach is fully experimental and a wide experimental campaign is carried out toward this aim. A series of accompanying papers will follow, one devoted to the numerical validation of the present experimental campaign, another presenting the experimentation carried out on different devices constituted by 
several layers of regenerated rubber where dry glass fiber layers are interposed, the last validating with advanced numerical modeling the experimentation carried out on the low-cost isolator prototypes.

Two types of regenerated EPDM (called type A and type $\mathrm{B}$ in the paper) are utilized in the experimental campaign and blended with two different virgin rubbers, namely Vistalon 3666 and Dutral 4038, producing 4 different rubber typologies. The first virgin rubber is used to produce a compound with a hardness of around 30 Shore A, while the latter with a hardness of 60 Shore A. A series of experimental tests are performed including rheometer characterization at different temperatures, Shore A hardness, compression set, uniaxial tensile tests and ageing characterization. The results show that the compounds with reactivated EPDM type B present the most satisfactory performance, before and after ageing.

This paper discusses also the method in fabricating FREIs, made of the selected recycled rubber and the glass fiber-reinforced polymer (GFRP). The hardness test performed on the sliced FREI specimen indicates that the vulcanization temperature used in the production is roughly suitable to obtain the expected rubber properties.

The novelty of the research, which is in this phase mainly experimental, stands in the design of small, low cost, unbonded recycled rubber seismic isolation devices to be used in developing countries (the main feature being the acceptable performance and the low cost) for low rise new masonry buildings $[9,10]$. It is worth mentioning that a suitable isolation system for such low rise buildings, which are typically characterized by a quite reduced $(15-20 \mathrm{~cm})$ thickness of the load-bearing walls requires the utilization of many isolators having small dimensions, and hence a hardness of the single pads higher than that used in common isolation, more similar to that adopted for bridge bearings. For this reason, one of the selection criteria among the different batches considered is certainly the higher hardness.

Further improvements of the idea here reported are going to be handled, such as a more quick and flexible preparation of the devices at an industrial level, their connection with foundation and superstructure to avoid possible rollover phenomena and the substitution of glass fibers with thermally more stable (possibly recycled) polymers.

\section{Experimental}

Four rubber batches using regenerated EPDM as shown in Fig. 1 are produced in this experimental campaign. Two commercial virgin rubbers Vistalon 3666 and Dutral 4038 are used to be blended with the regenerated EPDM to obtain blends with hardness $30 \pm 5 \mathrm{Sh} \mathrm{A}$ and $60 \pm 5 \mathrm{Sh} \mathrm{A}$. The first is a product with extended oil and medium/large distribution of molecular weight, an ENB content equal to $4.5 \%$ and a propylene content $30 \%$ by weight; the latter has a narrow/medium

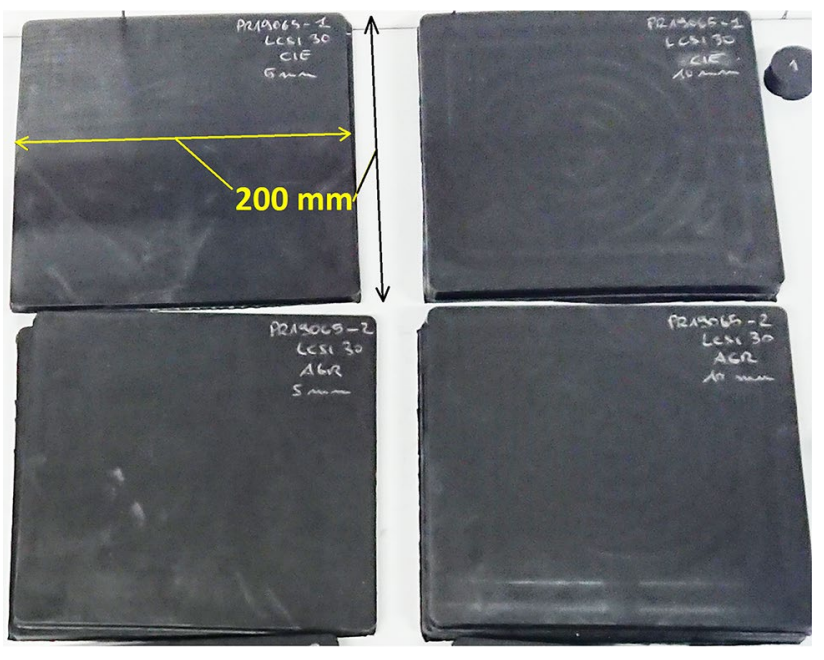

Fig. 1 Four recycled rubber specimens under study

molecular weight distribution, an ENB content of $4.1 \%$ and a propylene content of $29 \%$.

Vistalon 3666 exhibits a Mooney viscosity $(1+4)$ at $100{ }^{\circ} \mathrm{C}$ equal to 71 and at $121^{\circ} \mathrm{C}$ of 53; paraffinic oil content is high and equal to $42 \%$ by wt. Dutral 4038 exhibits a Mooney viscosity $(1+4)$ at $121^{\circ} \mathrm{C}$ equal to 65 . The detail composition of Vistalon 3666 and Dutral 4038 is reported in Table 1.

The detail composition of the 4 batches are presented in Table 2: batches 1 and 2 are designed as soft rubber with the target of hardness $30 \mathrm{Sh} \mathrm{A}$, while batches 3 and 4 are expected to have hardness of about $60 \mathrm{Sh} \mathrm{A}$. The regenerated EPDM is used to partially replace the virgin rubbers. As shown, the difference between batches 1 and 2 is the source of the regenerated EPDM: two sources A and B are considered. This difference applies also for batches 3 and 4 . As can be observed (comparing Table 2 with Table 1) the amount of carbon black $\mathrm{CC}$ remains essentially unchanged when compared with common virgin EPDM, demonstrating that regenerated rubber has a good capacity of crosslinking and cannot be regarded exclusively as a filler surrogate. This is not surprising, because the process of regeneration relies into the partial or almost total devulcanization of the rubber waste and its introduction in a blend with virgin rubber. Devulcanized rubber shows, therefore, almost the same (or slightly lower) capability to crosslink when compared with virgin rubber.

\section{Results and discussion}

\section{Rheometer test at four temperatures}

Rubbers in raw state must undergo a vulcanization process to crosslink the molecular chains and to improve the rubber properties. A rheometer measures the viscoelastic properties 
Table 1 Composition of Vistalon 3666 and Dutral 4038 two virgin rubbers

\begin{tabular}{lrrlr}
\hline Vistalon 3666 & & & Dutral 4038 \\
\cline { 1 - 2 } \cline { 5 - 5 } Product & Phr & & Product & Phr \\
\hline Vistalon 3666 & 175 & & Dutral 4038 & 100 \\
FEF N550 (carbon black) & 80 & & FEF N550 (carbon black) & 70 \\
Sillitin Z (silica) & 80 & & ZnO (zinc oxide) & 5 \\
Flexon 876 (paraffinic oil) & 120 & & Stearic acid & 1 \\
ZnO (zinc oxide) & 5 & & SRF (carbon black) & 40 \\
Sillitin Z (corpuscular & 0.5 & Paraffinic oil & 80 \\
silica) & & & \\
MBT & 1.5 & & Rodrtax 2 (zinc stearate) & 4.5 \\
TMTDS & 0.8 & & ZDBDC & 1 \\
ZDEDC & 0.8 & & S & 2 \\
DTDM & 2 & & 303.5 \\
Total & 465.6 & & Total &
\end{tabular}

DTDM, 4,4'-di-thio-di-morpholine-sulphur

SRF C.B., thioformaldehyde

TMTDS, tetra-methyl-thiuram-di-sulfide

ZDEDC, di-ethyl-di-thiocrbamate

ZDBDC, Zinc di-butyl-di-thiocarbamate

MBT, 2-mercaptobenzothiazole

Flexon 876, oil for EPDM extension (EASTM D 2226)

of rubber compounds during the vulcanization process. To evaluate the effect of different temperatures, the recycled rubber specimens in this study are subjected to rheometric test at four different temperatures: $150,160,170$ and $180^{\circ} \mathrm{C}$. Figure 2 presents the rheometric curves of the four batches at different temperatures. In Table 3 some important rheometric parameters are summarized.

When a specimen in the rheometer is heated under pressure, the viscosity drops and the torque decreases. The lowest torque recorded on the curve is called ML (Moment Lowest). It represents the stiffness of uncured rubber at a given temperature.

As the curing begins, the torque rises. The time $t_{\mathrm{s} 2}$ is the starting time of the test, when the torque has increased 2 units above ML value (the corresponding time is $t_{\mathrm{ML}}$ ). It represents the scorch time or at which point the curing actually starts. As the curing progresses, the torque increases further. The gradient depends on the compound and curing method used. Shortly thereafter, the torque reaches a maximum value and tends to be constant. The highest recorded torque on the curve is called MH (Moment Highest). Time from the start of the test to the point where $90 \%$ of the $\mathrm{MH}$ value is reached is called $t_{90}$. Such a description applies also for $t_{10}$ and $t_{50}$. As shown in Fig. 2 and Table 3, the increase of vulcanization temperature accelerates the vulcanization time. However, vulcanization at $150{ }^{\circ} \mathrm{C}$ seems to be the optimal one, because it results in the highest $\mathrm{MH}$.
On the other hand, the lowest $\mathrm{MH}$ is obtained in the vulcanization at $180{ }^{\circ} \mathrm{C}$. Figure 3 summarizes in graphs the characteristic times (in minutes) of the rheometer curves at $150{ }^{\circ} \mathrm{C}$ and $180{ }^{\circ} \mathrm{C}$ for the 4 batches, deducing data from rheometer curves of Fig. 2. Such characteristic times are $t_{\mathrm{ML}}$ (time at minimum torque), $t_{\mathrm{s} 2}$ (time at scorch i.e., incipient curing), $t_{50}$ (time at $50 \%$ of vulcanization, corresponding to a torque that is one half the maximum torque) and $\mathrm{t}_{90}$ (time at $90 \%$ of vulcanization). Obviously $t_{\mathrm{ML}}<t_{\mathrm{s} 2}<t_{50}<t_{90}$. On the horizontal axis, the ratio between polymer and regenerate rubber is represented, so that data on the left refer to Vistalon 3666, whereas data on the right to Dutral 4038. As can be observed, Vistalon crosslinks slightly faster than Dutral 4038 and regenerated rubber RRA (batches 1 and 3) seems less reactive than RRB (batches 2 and 4). These results suggest that RRB should be preferred to RRA for the preparation of the pads. As it will be shown later in the paper, batch 4 will be identified as the best one for the preparation of the isolation pads; this notwithstanding also batch 2 exhibits excellent viscoelastic characteristics, but with a slightly worst response after ageing.

\section{Shore A hardness}

Shore (durometer) hardness is a measure of the resistance of a material towards the penetration of a spring-loaded needlelike indenter. In rubbers, the hardness is usually measured by shore A scales. In a rubber test for seismic isolation purpose, the hardness is measured before and after accelerated ageing. The accelerated aging is conditioned by storing the specimens in a oven at $70{ }^{\circ} \mathrm{C}$ for $24 \times 7 \mathrm{~h}$. The aged specimens are then tested after $24 \mathrm{~h}$ of storage at room temperature. According to EN 15129 [18], the variation of the hardness after aging is recommended not to exceed -5 or +8 . Table 4 presents the measured hardness of the four recycled rubber batches before and after aging and the hardness of the virgin rubbers provided by the supplier. Batches $3 \mathrm{~A}$ and $4 \mathrm{~B}$ satisfy the maximum variation of rubber hardness after accelerated aging.

\section{Compression set}

In compression set testing, the ability of rubber to return to its original thickness after prolonged compression at defined temperature and deflection is examined. When the rubber is compressed over time, it loses its ability to return to its original thickness. This loss of resilience may degrade the performance of rubber-based equipment such as seals or elastomeric gaskets. Compressions set results are expressed in a percentage. A rubber which exhibits a lower percentage has better resistance to permanent deformation under a defined deflection and temperature range. In the compression set, the rubber specimens as shown in Fig. 4 are subjected 
Table 2 Composition of 4 rubber specimens

\begin{tabular}{|c|c|c|c|c|c|c|c|}
\hline \multicolumn{2}{|l|}{ Batch 1A } & \multicolumn{2}{|l|}{ Batch 2B } & \multicolumn{2}{|l|}{ Batch 3A } & \multicolumn{2}{|l|}{ Batch 4B } \\
\hline Ingredient & $\mathrm{gr}$ & Ingredient & gr & Ingredient & gr & Ingredient & gr \\
\hline EPDM Vistalon 3666 OG & 175.00 & EPDM Vistalon 3666 OG & 175.00 & EPDM Dutral 4038 NCS & 100.00 & EPDM Dutral 4038 NCS & 100.00 \\
\hline EPDM Regenerated A & 300.00 & EPDM Regenerated B & 300.00 & EPDM Regenerated A & 300.00 & EPDM Regenerated B & 300.00 \\
\hline Zinc oxide & 9.52 & Zinc oxide & 9.52 & & & & \\
\hline Stearic acid & 1.52 & Stearic acid & 1.52 & & & & \\
\hline PEG 4000 & 4.00 & PEG 4000 & 4.00 & Zinc oxide & 4.00 & Zinc oxide & 4.00 \\
\hline $\begin{array}{l}\text { Polyethylene low molecu- } \\
\text { lar weight }\end{array}$ & 5.00 & $\begin{array}{l}\text { Polyethylene low molecu- } \\
\text { lar weight }\end{array}$ & 5.00 & Stearic acid & 1.00 & Stearic acid & 1.00 \\
\hline Sillitin N 85 & 68.00 & Stllitin N 85 & 68.00 & & & & \\
\hline Calcium carbonate & 100.00 & Calcium carbonate & 100.00 & Calcium carbonate & 40.00 & Calcium carbonate & 40.00 \\
\hline $\begin{array}{l}\text { N550 FEF II (carbon } \\
\text { black) }\end{array}$ & 72.80 & $\begin{array}{l}\text { N550 FEF II (carbon } \\
\text { black) }\end{array}$ & 72.80 & $\begin{array}{l}\text { N550 FEF II (carbon } \\
\text { black) }\end{array}$ & 185.00 & $\begin{array}{l}\text { N550 FEF II (carbon } \\
\text { black) }\end{array}$ & 185.00 \\
\hline Paraffinicoil & 142.00 & Paraffinic oil & 142.00 & Paraffinic oil & 95.00 & Paraffinic oil & 95.00 \\
\hline MBT premix & 1.92 & MBT premix & 1.92 & & & & \\
\hline ZDBC premix & 1.92 & ZDBC premix & 1.92 & & & & \\
\hline TDEC premix & 0.80 & TDEC premix & 0.80 & MBT premix & 1.50 & MBT premix & 1.50 \\
\hline TMTD premix & 1.12 & TMTD premix & 1.12 & Sulphur premix & 2.50 & Sulphur premix & 2.50 \\
\hline DPTT premix & 1.12 & DPTT premix & 1.12 & TMTD premix & 2.00 & TMTD premix & 2.00 \\
\hline Sulphur premix & 3.80 & Sulphur premix X & 3.80 & & & & \\
\hline Total & 888.52 & Total & 888.52 & Total & 731.00 & Total & 731.00 \\
\hline
\end{tabular}

DPTT, dipentamethylene thiuram tetrasulfide

EG 4000, polyethylene glycol

SILLITIN N 85, silica and lamellar kaolinite

MBT, 2-mercaptobenzothiazole

N550 FEF, carbon black N 550

PEG 4000, polyethylene glycol

TDEC, tellurium diethyl dithiocarbamate

TMTD, tetramethyl thiuram disulfide

ZDBC, zinc dibutyl dithiocarbamate

to compression at $70{ }^{\circ} \mathrm{C}$ for $24 \mathrm{~h}$. According to EN 15129 [18] in case of rubber material for seismic isolation, the maximum value of compression set result is $30 \%$. In the present experimental test, the results of the compression set of rubber batches $1 \mathrm{~A}, 2 \mathrm{~B}, 3 \mathrm{~A}$, and $4 \mathrm{~B}$ are respectively the following: $23,12,28$, and $25 \%$. Therefore, all four compounds satisfy the requirement of compression set for rubber seismic isolation.

\section{Uniaxial tensile test}

Rubber is well-known as an ideal example of perfectly elastic material. However, nonlinear elasticity of rubber at moderate to large strain is clearly remarkable. Such a nonlinearity is often called as hyperelasticity. To characterize the hyperelastic properties of the proposed recycled rubber, a uniaxial test based on ISO 37 [19] is performed in this study.
For each rubber batch, three specimens in the form of dumb-bell pieces as shown in Fig. 5 are tested in the uniaxial tensile test device. The specimens are stretched to the extent of failure to define the tensile strength and the strain at failure.

Figure 6 presents the results of the uniaxial tensile tests on the four rubber batches. A single curve represents the average of three identical specimens. Results from the test of fresh specimens are presented by black curves, while the red curves present the results after aging. The accelerated aging is conditioned by storing the specimens in a oven at $70{ }^{\circ} \mathrm{C}$ for $24 \times 7 \mathrm{~h}$. The aged specimens are then tested after $24 \mathrm{~h}$ of storage at room temperature.

In the case of unaged soft rubber compounds, batch 1 exhibits larger failure strain yet lower tensile strength when compared to batch 2 . Both soft batches present an identical shape of the hyperelastic curves. For hard rubber compounds, batch 3 experiences larger failure strain yet lower tensile strength compared to batch 4 . The tensile strength of 

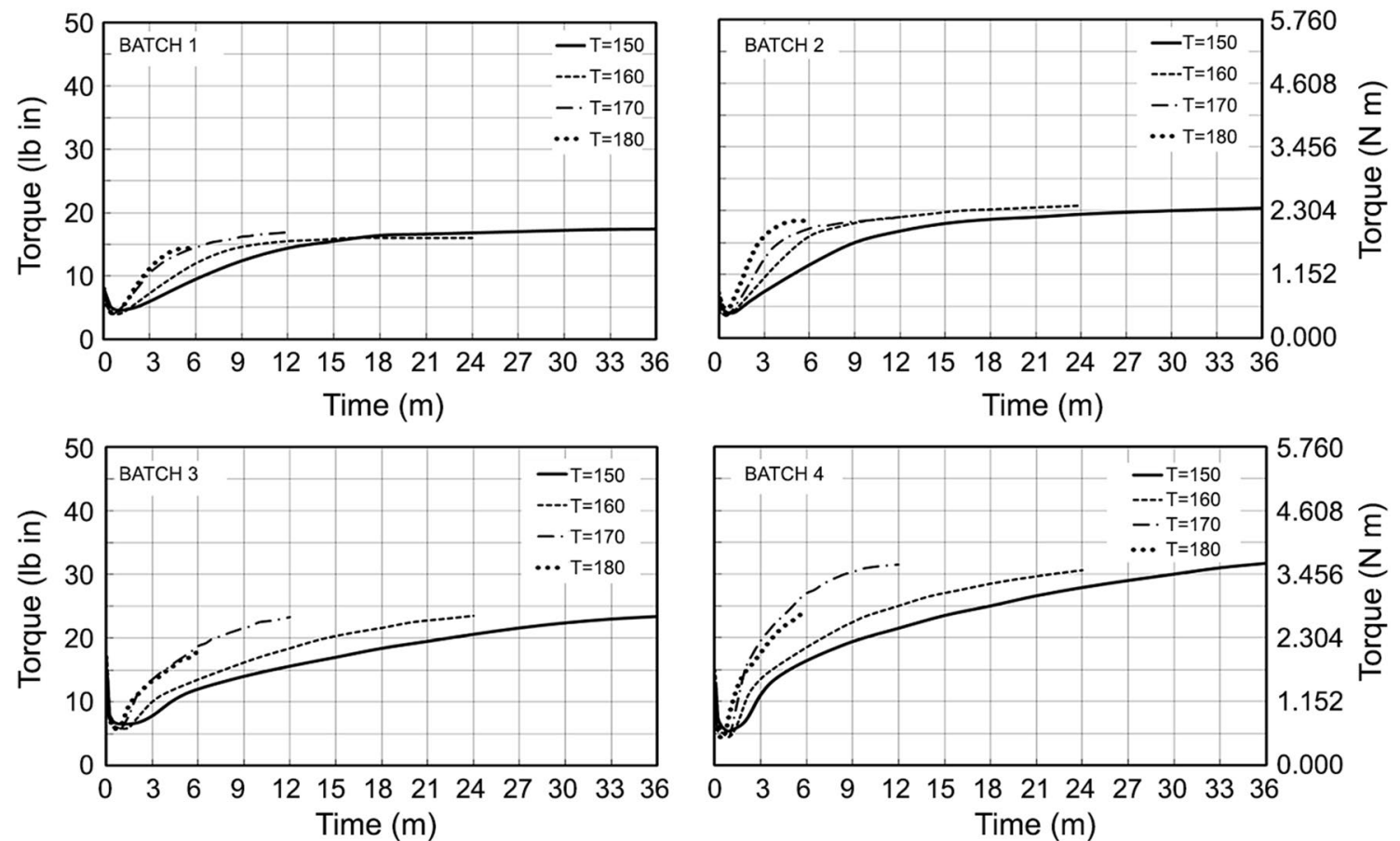

Fig. 2 Rheometer curves of 4 rubber specimens at different temperatures

Table 3 Several parameters obtained in the rheometer test on 4 rubber specimens at different temperatures

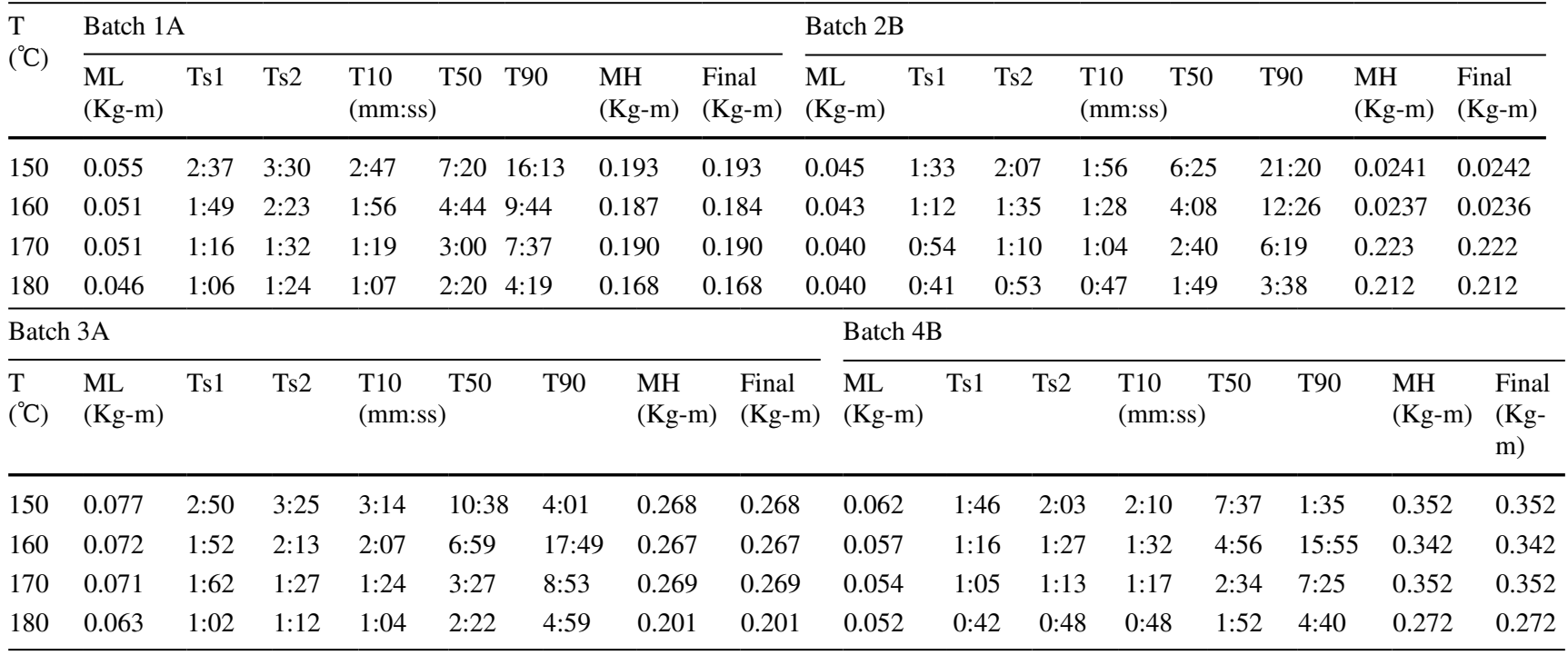

the 4 batches varies from 5.4 to $6.5 \mathrm{MPa}$, while the failure strain ranges from 310 to $840 \mathrm{MPa}$. In general, the values of tensile strength of 4 batches under study are significantly lower than the requirements stated in EN 15129 [18] for commercial seismic isolators. However, the proposed recycled rubbers are intended to be used for unbonded isolators which exhibit much lower tensile stress under large shear displacement when compared to the commercial ones, as reported in the literature [20].

In Fig. 6, the results of tensile tests after aging are presented by red curves. In general, the aging increases the stiffness of the rubber. Table 5 shows the quantity of difference of tensile strength and strain-at-failure, before and after aging. According to EN 15129 [18], the maximum 

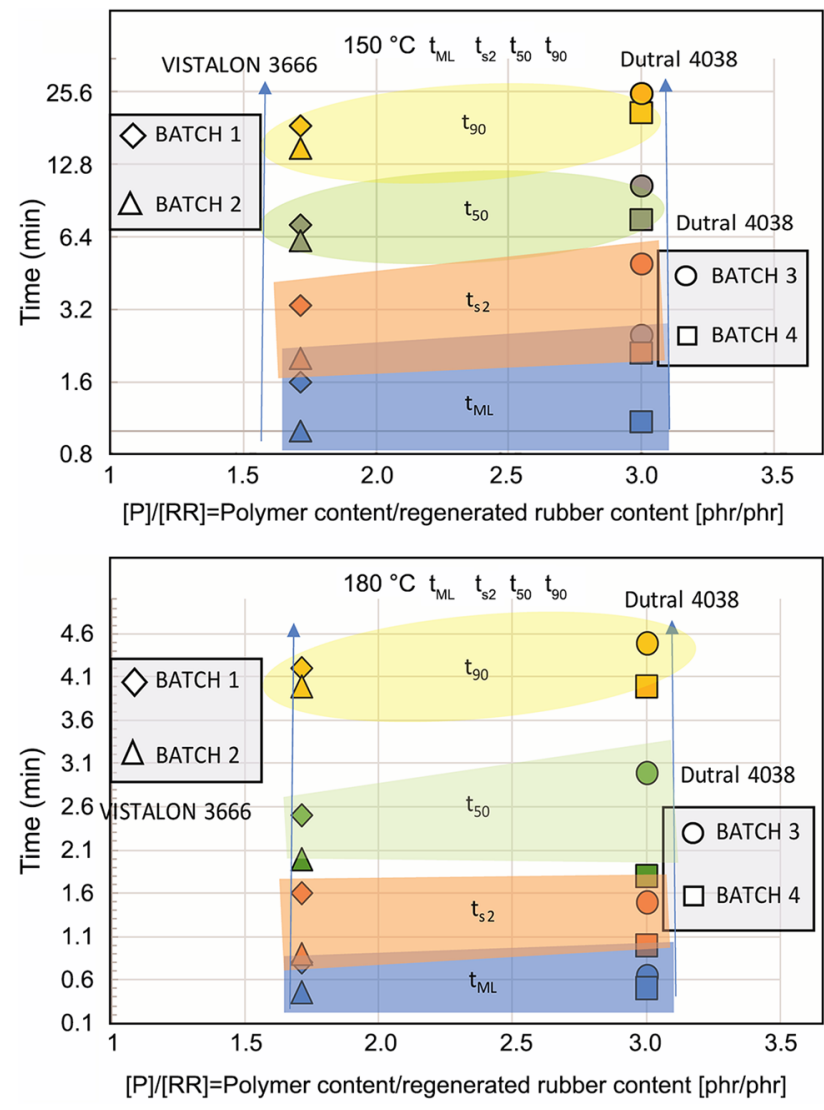

Fig. 3 Characteristic times of the 4 batches analyzed as a function of the content ratio between virgin polymer and regenerated rubber

Table 4 Shore A hardness of 4 recycled rubber specimens before and after aging

\begin{tabular}{llll}
\hline Rubber & \multicolumn{2}{l}{ Shore A hardness } & $\Delta \mathrm{H}$ \\
\cline { 2 - 3 } & Unaged & Aged & \\
\hline Vistalon 3666 $^{\mathrm{a}}$ & 34 & - & - \\
Batch 1A & 27 & 37 & 10 \\
Batch 2B & 33 & 43 & 10 \\
Dutral 4038 & 55 & - & - \\
Batch 3A & 56 & 62 & 6 \\
Batch 4B & 66 & 69 & 3 \\
\hline
\end{tabular}

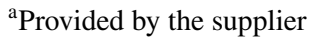

changes of tensile strength and failure strain are $15 \%$ and $25 \%$, respectively. Therefore, batches $2 \mathrm{~B}$ and $4 \mathrm{~B}$ satisfy the aging test requirements in terms of tensile properties.

Figure 7 summarizes the experimental results obtained for the different receipts investigated at failure. On the horizontal axis the elongation-at-break (EB) in \% is represented, whereas on the vertical axis the ultimate strength (TS) in $\mathrm{MPa}$ is depicted. The corresponding data of two virgin rubbers Vistalon 3666 and Dutral 4038 and 4 mixed regenerated rubbers are presented. In this way, the comparison with the reference compound made exclusively by virgin rubber is straightforward.

From Fig. 7, it can be deduced that the different formulations with regenerated rubber, having comparable hardness with those of the virgin materials, generally exhibit a lower ultimate strength. However, blends with type B regenerated rubber (RRB) exhibits a much better performance than type A regenerated rubber (RRA). As expected, adding regenerated rubber results into a decrease of the strength with similar elongation-at-break.

In Fig. 8, the diagrams of the tensile strength (TS) as a function of ultimate elongation-at-break (EB) after ageing at $70{ }^{\circ} \mathrm{C}$ for $128 \mathrm{~h}$ are presented. From Fig. 8 , it is possible to notice that by ageing the elongation-at-break decreases and the tensile strength slightly increases. Considering the theoretical behavior of the observed EB and TS as a function of the vulcanization degree depicted in Fig. 9b, it can be argued that the compounds are slightly under-vulcanized. From a chemical point of view, ageing has the effect to promote an additional crosslinking, confirmed by the fact that Shore A (Hs) also increases (Fig. 8). Ageing results, therefore, into an increase of the vulcanization degree i.e., non-cured polymer that is present at the end of the vulcanization process reticulates further during ageing. The slight increase of the tensile strength shows how the initial curing condition is, however, near the optimal one i.e., not far from the point of maximum obtainable (Fig. 8).

Figure 9a shows that compound 4B (Dutral $4038+$ regenerated rubber $B$ ) is that one exhibiting the best performances among all those investigated. The crosslinking density appears slightly suboptimal, because TS after aging exhibits a moderate increase, whereas EB decreases roughly from $300 \%$ to $260 \%$. To be quantitatively conclusive about the most suitable vulcanization conditions imposed during the production phase, the authors are planning to develop ad-hoc kinetic numerical models, which first of all should account for reversion phenomenon in a rheometer chamber [21], then should try to help in the optimization of a real industrial production process with finite elements (FEs) [22, 23] and finally should perform automatic back analyses (assuming as objective function some expected mechanical properties), to determine the optimal production parameters by means of innovative and fast meta-heuristic approaches $[24,25]$. As a matter of fact, compound $4 \mathrm{~B}$ is the blend approximating better the behavior of the virgin material. Values of EB and TS for Dutral 4038 are scaled in the figure by a factor $26 / 33$, because in the blends a Dutral 4038 with $26 \%$ in weight of polymer was utilized, whereas data furnished by the producer (Table 1), for the virgin material refer to an amount of polymer equal to $33 \%$. 
Fig. 4 a Rubber specimens for compression set; $\mathbf{b}$ standard device for compression set
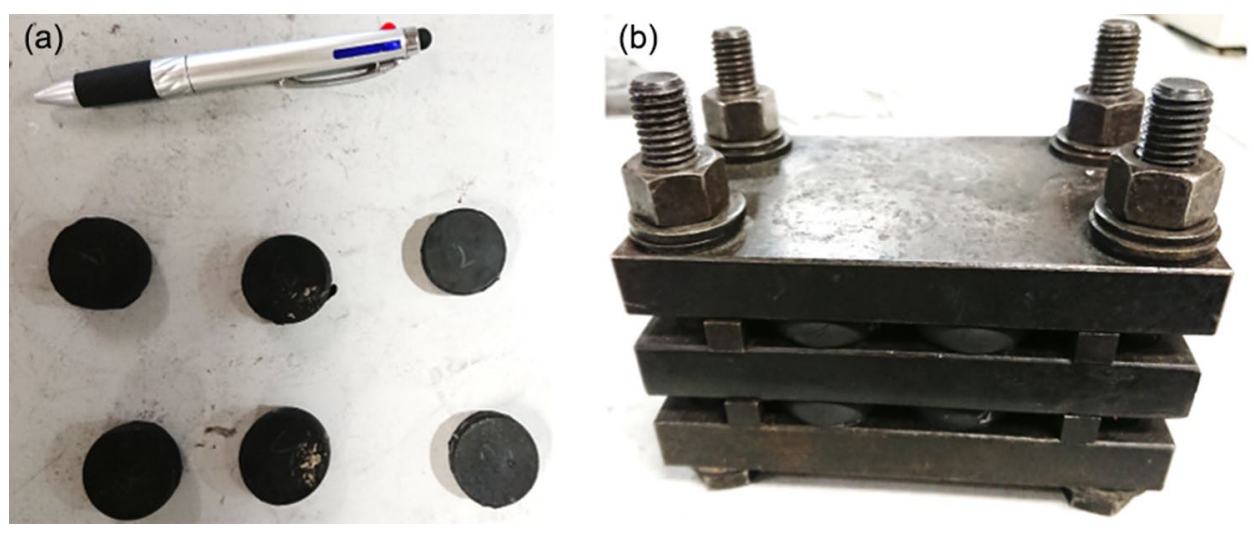

Fig. 5 Dumb-bell specimens of rubber and the uniaxial tensile test device
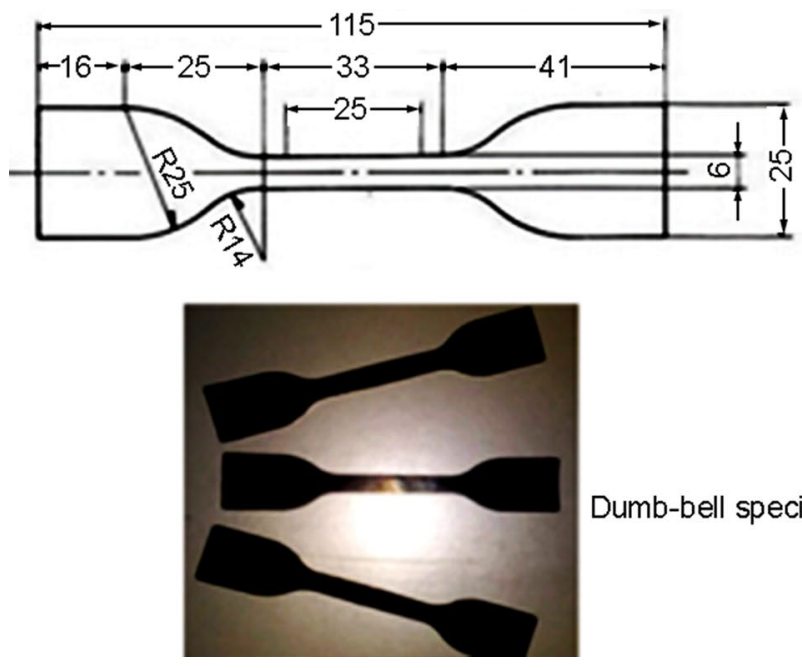

Dumb-bell specimen

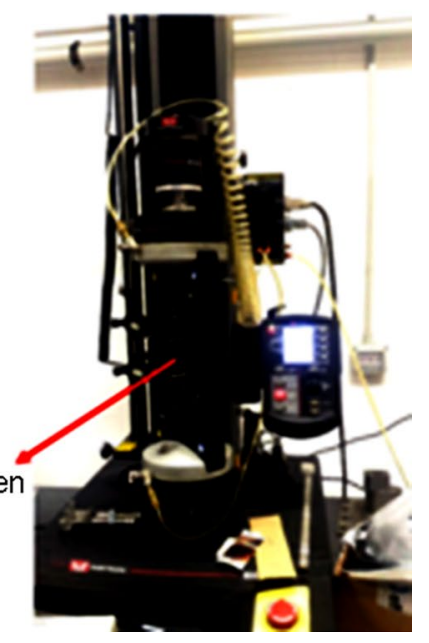

Fig. 6 Strain stress curves of four rubber batches before and after aging
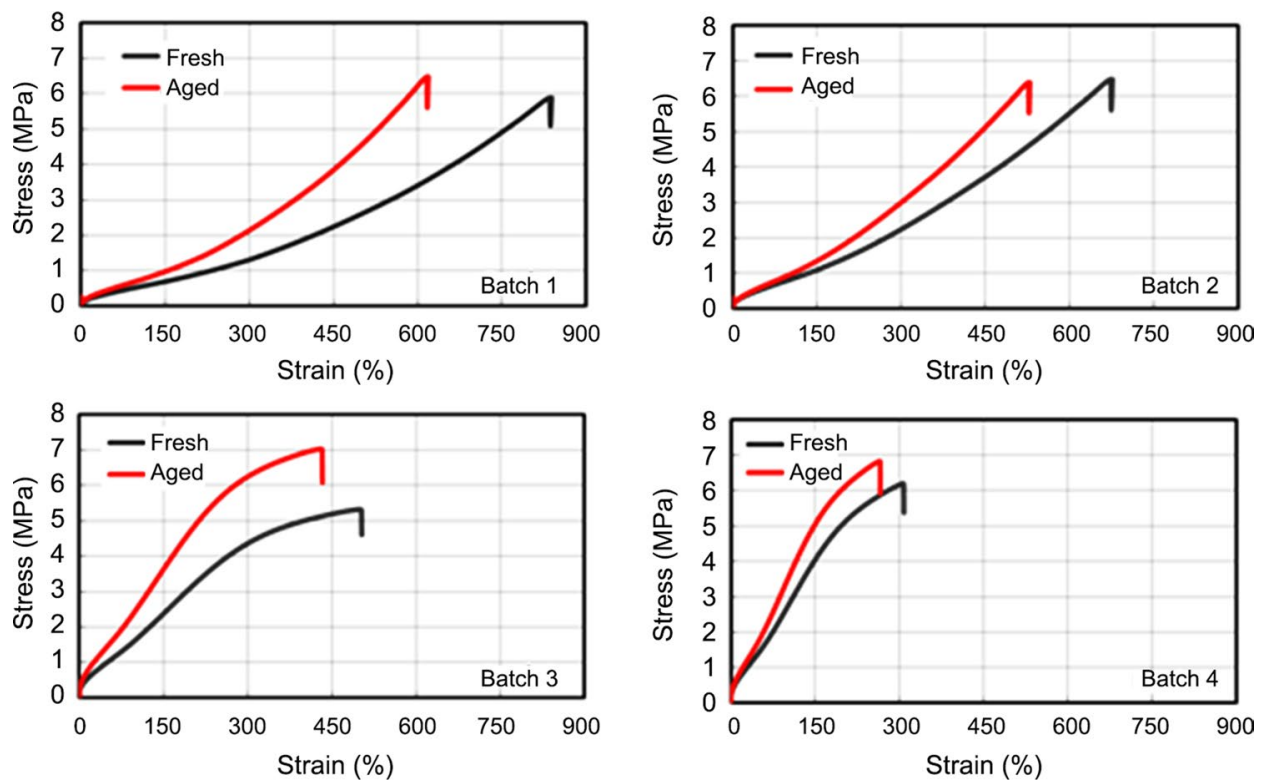
Table 5 Tensile strength and failure strain of 4 recycled rubber specimens before and after aging

\begin{tabular}{|c|c|c|c|c|c|c|}
\hline & \multicolumn{2}{|c|}{ Tensile strength (MPa) } & \multirow[t]{2}{*}{$\Delta(\%)$} & \multicolumn{2}{|c|}{ Strain-at-break (\%) } & \multirow[t]{2}{*}{$\Delta(\%)$} \\
\hline & Unaged & Aged & & Unaged & Aged & \\
\hline Vistalon $3666^{\mathrm{a}}$ & 9.3 & - & - & 730 & - & - \\
\hline Batch 1A & 5.89 & 6.48 & 10.01 & 839 & 619 & -26.22 \\
\hline Batch 2B & 6.48 & 6.39 & -1.38 & 676 & 529 & -21.74 \\
\hline Dutral $4038^{\mathrm{a}}$ & 11 & - & - & 400 & - & - \\
\hline Batch 3A & 5.32 & 7.03 & 32.14 & 503 & 433 & -13.91 \\
\hline Batch 4B & 6.2 & 6.83 & 10.16 & 308 & 266 & -13.63 \\
\hline
\end{tabular}

${ }^{\text {a}}$ Provided by the producer
Fig. 7 Comparison between first group (red colors: Vistalon $3666,1 \mathrm{~A}$ and $2 \mathrm{~B}$ ) and the second group (blue colors: Dutral 4038, 3A and 4B): elongationat-break (EB) and tensile strength (TS)

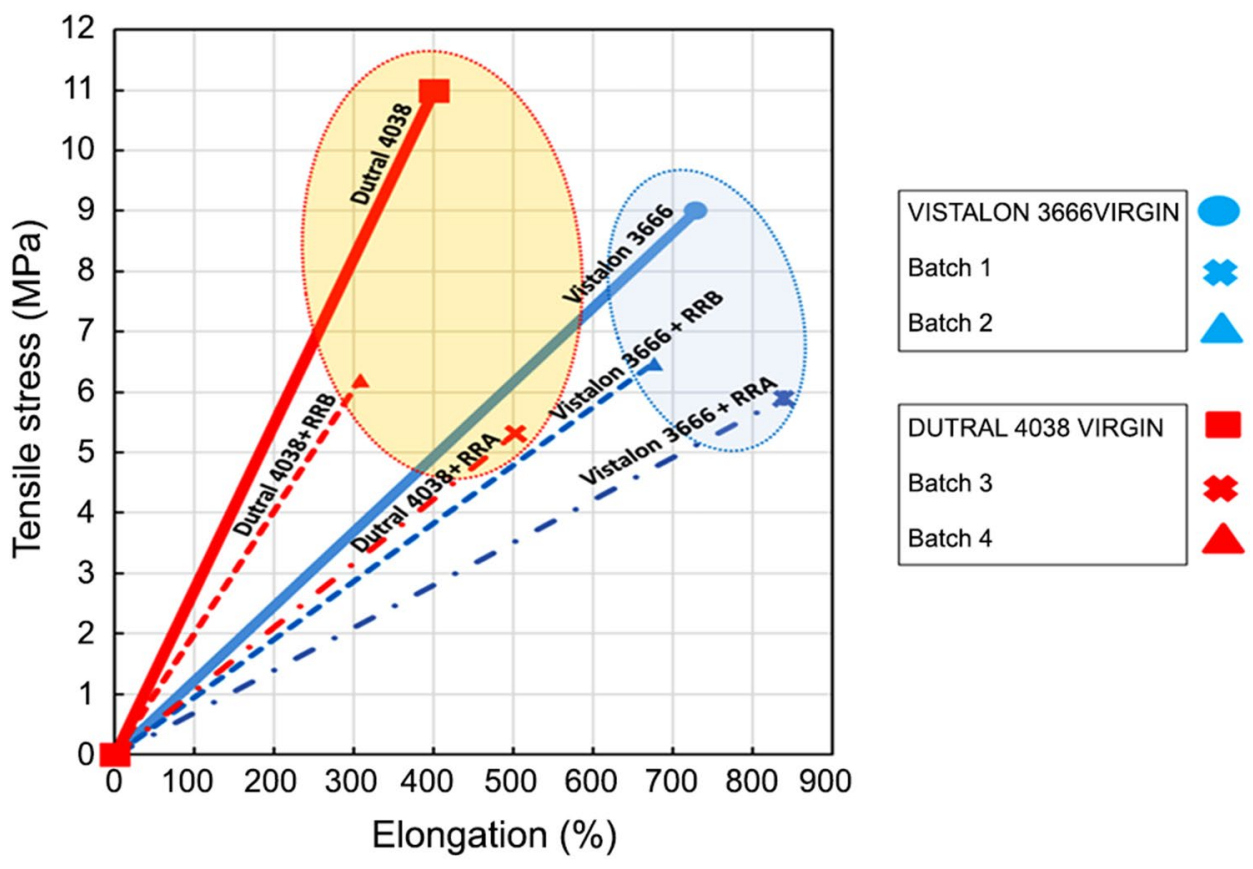

It is finally worth mentioning that quite satisfactory is also the behavior of blend 2B (regenerated rubber with Vistalon 3666), but with a still slightly better performance of $4 \mathrm{~B}$.

\section{Production of fiber-reinforced elastomeric isolators (FREIs)}

After having obtained the rubber compound with the best performance, the fiber reinforcements and the vulcanization devices are prepared. Pre-treated woven glass fibers are used in this project. The fibers are dried with a primer to improve adhesion i.e., chemically gripped (Fig. 10). The product is a commercial cover-coat adhesive (Megum 538 by Dupont), used in combination with an adhesive primer (Thixon by Dupont), commonly adopted for bonding rubber compounds to metals and other rigid substrates during vulcanization.

Figure 11a presents the preparation of rubber pads and fiber reinforcements having dimension $75 \times 75 \mathrm{~mm}$ to be vulcanized in the mold (Fig. 11b). The vulcanization is performed at $150{ }^{\circ} \mathrm{C}$ for 40 min by compression molding. Typically the isolators are constituted by 4-5 layers of GFRP, as indicated by the cross-section in Fig. 11c. After the vulcanization, to evaluate the quality of the production process, the Shore A hardness is measured in the middle vertical section after knife cut, as can be noted in Fig. 11d. Some tribology damage of the cut surface is observed near the boundary for the utilization of a toothed knife. Five hardness measures were reported, four points near the section corners (points 2, 3, 4 and 5) and one in the center. A Shore A hardness of $58 \pm 2$ corresponding to the corners and of $48 \pm 2$ in the center is reported during measurement. On the other hand, a rubber cube without fiber sheets exhibits a homogeneous hardness from the skin to the core of $62 \pm 2 \mathrm{Sh}$ A.

Considering that an analogic durometer is used and due to non-flat and damaged surface obtained, the following considerations can be drawn: 

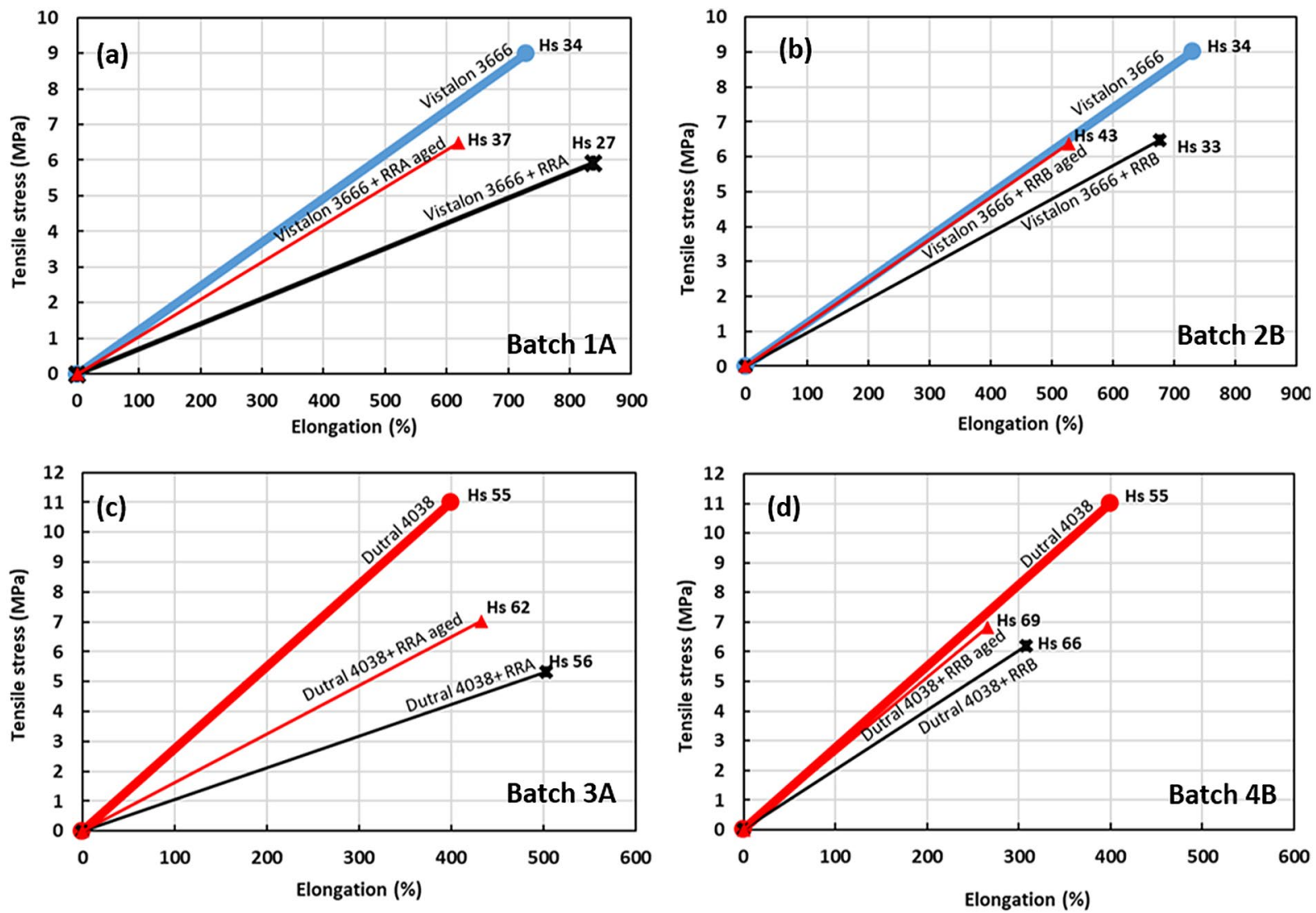

Fig. 8 Comparison between virgin and regenerated rubbers, regarding the elongation-at-break (EB) and tensile strength (TS), in aged and unaged conditions: a Vistalon 3666 and compound 1A; b Vistalon

3666 and compound 2B; b Dutral 4038 and compound 3A; and d Dutral 4038 and compound 4B
Fig. 9 a Scaled Dutral 4038 values and 4B values (with and without ageing) comparison in terms of elongation-at-break (EB) and tensile strength (TS). b theoretical behavior of $\mathrm{EB}$ and TS as a function of the vulcanization degree

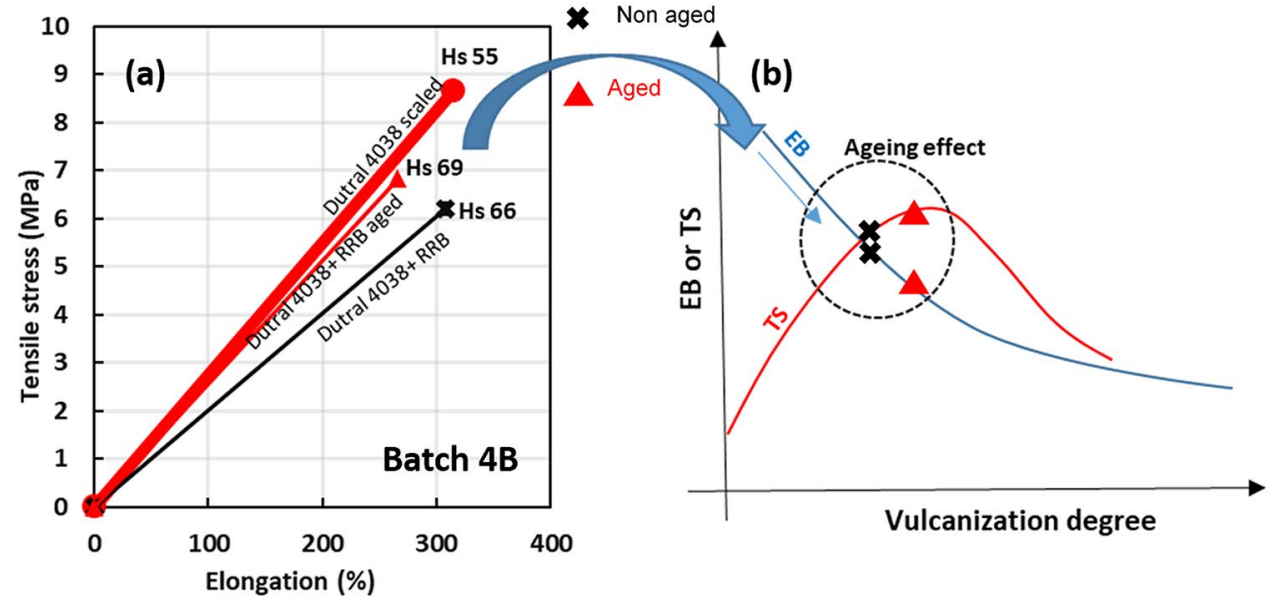

- Mold and vulcanization temperature $\left(150{ }^{\circ} \mathrm{C}\right)$ are roughly suitable to obtain industrial items vulcanized in a proper and uniform manner.

- The lower hardness registered in correspondence of the center of the section confirms that the presence of
GFRP sheets is detrimental, since it isolates further the core of the samples.

- Final hardness is acceptable both in the core and near the skin. 
Fig. 10 Megum 538 used in combination with Thixon for adhesive bonding between GFRP and rubber pads
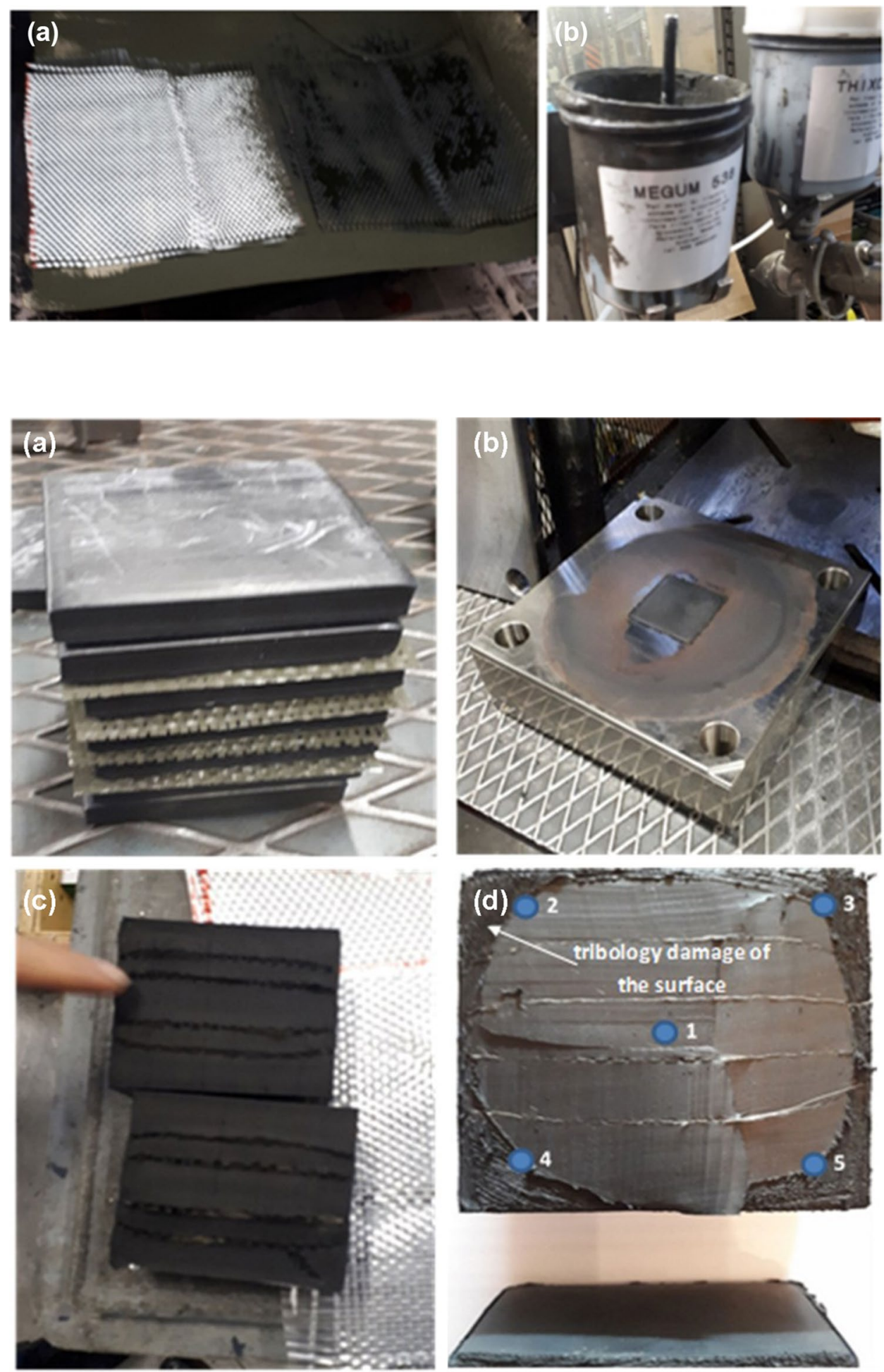

Fig. 11 a un-vulcanized pads to insert in the mold; $\mathbf{b}$ steel mold designed to produce seismic isolator prototypes with $75 \mathrm{~mm}$ edge length; $\mathbf{c}$ first samples produced and cut in the middle (central, top and bottom pads are thicker on purpose); and $\mathbf{d}$ points where the hardness is measured on the vertical central cut section

\section{Conclusions}

A series of experimental tests on 4 different rubber compounds made of reactivated EPDM have been carried out. Two commercial virgin rubbers Vistalon 3666 and Dutral 4038 have been used to be blended with regenerated
EPDM to obtain blends with hardness $30 \pm 5 \mathrm{ShA}$ and $60 \pm 5$ Sh A. Two different sources of reactivated EDPM (types A and B) have been considered to find the best receipt of the compound. Aging effect has been also investigated to evaluate the durability of the rubbers. 
Regarding rheometer tests, authors conclude that the vulcanization at $150{ }^{\circ} \mathrm{C}$ seems to be the most suitable temperature, being associated with the highest $\mathrm{MH}$ obtained (moment highest) when compared to other vulcanization temperatures. From the hardness test, it is found that compounds labeled as $3 \mathrm{~A}$ and $4 \mathrm{~B}$ satisfy the requirement of the hardness variation before and after aging, indicated by their hardness variation after ageing below the range -5 to +8 Sh A. In the compression set test, all 4 compounds satisfied the requirement of compression set for rubber seismic isolators, where all the thickness variations after test were less than $30 \%$.

In the uniaxial tensile test, the performance of 4 compounds containing regenerated EDPM has been also compared to the virgin rubbers. It has been found that the different formulations with regenerated EPDM, having comparable hardness with that of the virgin materials, generally exhibited a tensile strength lower than the virgin ones. However, blends with type B regenerated rubber exhibited a much better performance than type A regenerated rubber. As expected, adding regenerated rubber resulted into a decrease of the strength with similar elongation-at-break. In the ageing state, the elongation-at-break decreased and the tensile strength slightly increased. A slight increase of hardness after ageing was observed, indicating that additional crosslinking occurred during ageing process, increasing the vulcanization degree of the compound.

Compound 4B (Dutral 4038 + regenerated EPDM type B) resulted as the most satisfactory one before and after aging. It approximated better the performance of the corresponding virgin material, Dutral 4038. Quite satisfactory results were also obtained by compound 2B (Vistalon 3666 + regenerated EPDM type B), which exhibited allowable variation of both tensile strength and elongation-at-break after ageing.

In the production of the fiber-reinforced elastomeric isolators (FREIs), the bond between GFRP and rubber pad was provided by a cover-coat adhesive used in combination with an adhesive primer applied directly on the dry fiber. After the vulcanization, hardness tests were performed at several points on the sliced FREI specimen. The shore A hardness at the middle of the pad was found to be considerably lower than that measured near the edge of the pad. On the other hand, a rubber cube without GFRP presented an identical hardness at any point, confirming that the presence of GFRP sheets isolates further the core of the samples, being, therefore, a negative feature to take carefully into account in the production process.

Finally, it is worth underlying that the aim of the research presented in this paper has been to trace roughly a first tangible possibility to regenerate previously vulcanized items in EPDM in the production of new devices for useful civil engineering applications, maintaining the production cost sufficiently low. The research belongs to a wider project aimed at the production of low-cost seismic isolation devices, which is still ongoing and the results will be made available to the scientific community in the near future. A comprehensive experimental campaign is taking place at the Technical University of Milan (Italy), which includes the determination of damping properties of the pads, dynamic modulus, hysteretic behavior on both isolated pads and entire device, in both un-bonded and bonded conditions, before and after ageing.

Funding Open access funding provided by Politecnico di Milano within the CRUI-CARE Agreement.

Open Access This article is licensed under a Creative Commons Attribution 4.0 International License, which permits use, sharing, adaptation, distribution and reproduction in any medium or format, as long as you give appropriate credit to the original author(s) and the source, provide a link to the Creative Commons licence, and indicate if changes were made. The images or other third party material in this article are included in the article's Creative Commons licence, unless indicated otherwise in a credit line to the material. If material is not included in the article's Creative Commons licence and your intended use is not permitted by statutory regulation or exceeds the permitted use, you will need to obtain permission directly from the copyright holder. To view a copy of this licence, visit http://creativecommons.org/licenses/by/4.0/.

\section{References}

1. Coates A (2015) The commerce in rubber: the first 250 years. Oxford University, Oxford

2. Morawetz H (2000) History of rubber research. Rubber Chem Technol 73:405-426

3. Guise-Richardson C (2010) Redefining vulcanization: Charles goodyear, patents, and industrial control, 1834-1865. Technol Cult 51:357-387

4. Gregori A, Castoro C, Marano GC, Greco R (2019) Strength reduction factor of concrete with recycled rubber aggregates from tires. J Mater Civil Eng 31:04019146

5. Mendis AS, Al-Deen S, Ashraf M (2017) Effect of rubber particles on the flexural behaviour of reinforced crumbed rubber concrete beams. Constr Build Mater 154:644-657

6. Mendis AS, Al-Deen S, Ashraf M (2018) Flexural shear behaviour of reinforced Crumbed Rubber Concrete beam. Constr Build Mater 166:779-791

7. Tsang HH, Lo SH, Xu X, Neaz Sheikh M (2012) Seismic isolation for low-to-medium-rise buildings using granulated rubber-soil mixtures: numerical study. Earthq Eng Struct Dyn 41:2009-2024

8. Spizzuoco M, Calabrese A, Serino G (2014) Innovative low-cost recycled rubber-fiber-reinforced isolator: experimental tests and finite element analyses. Eng Struct 76:99-111

9. Habieb AB, Valente M, Milani G (2019) Base seismic isolation of a historical masonry church using fiber-reinforced elastomeric isolators. Soil Dyn Earthq Eng 120:127-145

10. Habieb AB, Milani G, Tavio T (2018) Two-step advanced numerical approach for the design of low-cost unbonded fiber-reinforced elastomeric seismic isolation systems in new masonry buildings. Eng Fail Anal 90:380-396

11. De Raaf MGP, Tait MJ, Toopchi-Nezhad H (2011) Stability of fiber-reinforced elastomeric bearings in an unbonded application. J Compos Mater 45:1873-1884 
12. Calabrese A, Spizzuoco MC, Serino G, Della Corte G, Maddaloni G (2015) Shaking table investigation of a novel, low-cost, base isolation technology using recycled rubber. Struct Control Hlth 22:107-122

13. Das A, Deb SK, Dutta A (2016) Shake table testing of un-reinforced brick masonry building test model isolated by U-FREI. Earthq Eng Struct Dyn 45:253-272

14. Maddaloni G, Caterino N, Occhiuzzi A (2017) Shake table investigation of a structure isolated by recycled rubber devices and magnetorheological dampers. Struct Control Hlth 24:e1906

15. Mishra HK, Igarashi A, Matsushima H (2013) Finite element analysis and experimental verification of the scrap tire rubber pad isolator. Bull Earthq Eng 11:687-707

16. Turer A, Özden B (2008) Seismic base isolation using low-cost Scrap Tire Pads (STP). Mater Struct 41:891-908

17. Morales E, Filiatrault A, Aref A (2017) Sustainable and low cost room seismic isolation for essential care units of hospitals in developing countries. In 16th World Conference on Earthquake Engineering. Santiago

18. EN 15129 (2009) Antiseismic Devices. Brussels: Comité Européen de Normalisation (CEN)

19. ISO 37(2005) Rubber vulcanized or thermoplastic determination of tensile stress-strain properties. International Organization for Standardization: Geneva
20. Toopchi-Nezhad H, Tait MJ, Drysdale RG (2011) Bonded versus unbonded strip fiber-reinforced elastomeric isolators: finite element analysis. Compos Struct 93:850-859

21. Milani G, Milani F (2011) A three-function numerical model for the prediction of vulcanization-reversion of rubber during sulfur curing. J Appl Polym Sci 119:419-437

22. Milani G, Milani F (2012) A comprehensive numerical model for the interpretation of crosslinking with peroxides and sulphur: chemical mechanisms and optimal vulcanization of real items. Rubber Chem Technol 85:590-628

23. Milani G, Milani F (2010) Optimal vulcanization of 2D-3D EPM/ EPDM thick elements through peroxidic mixtures. J Math Chem 47:229-267

24. Milani G, Milani F (2009) Optimization of power cable production lines for EPM/EPDM elastomers by genetic algorithm with different peroxides. J Appl Polym Sci 11:482-507

25. Milani G, Milani F (2008) Genetic algorithm for the optimization of rubber insulated high voltage power cables production lines. Comput Chem Eng 32:3198-3212 